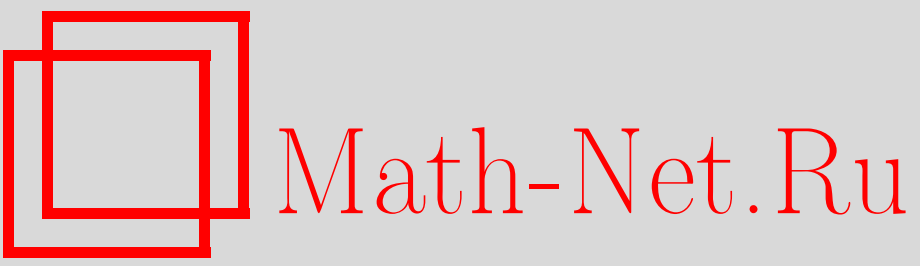

М. Е. Вейсман, С. Ю. Калмыков, Коррелированные когерентные состояния двумерного квантового осциллятора с нестационарной связью мод, ТМФ, 1997, том 111, номер 2, 304-311

DOI: https://doi.org/10.4213/tmf1009

Использование Общероссийского математического портала Math-Net.Ru подразумевает, что вы прочитали и согласны с пользовательским соглашением

http://www.mathnet.ru/rus/agreement

Параметры загрузки:

IP: 54.224 .60 .19

26 апреля 2023 г., 18:04:00 


\section{КОРРЕЛИРОВАННЫЕ КОГЕРЕНТНЫЕ СОСТОЯНИЯ ДВУМЕРНОГО КВАНТОВОГО ОСЦИЛЛЯТОРА С НЕСТАЦИОНАРНОЙ СВЯЗЬЮ МОД}

Исследованы явления сжатия и корреляции квантовых фолуктуаций в коррелированных когерентных состояниях системы двух осцилляторных мод с параметрической связью координат. В условиях сильной связи мод обнаружены возбуждение и сильная корреляция квантовых флуктуаций. Матрица дисперсий и ковариаций импульсов и координат найдена в явном виде с помощью метода линейных квантовых интегралов движения.

1. Двумерный квантовый осциллятор с параметрической связью мод может служить моделью двухмодового сжатого света $[1,2]$, интерес к статистическим свойствам которого связан с использованием параметрического возбуждения в разнообразных квантовооптических системах [3] с целью получения сжатых [4] и коррелированных [5] состояний электромагнитного поля. В недавних работах [6-8] исследовались параметрическое $[6,7]$ и нелинейное [8] возбуждения бозе-осцилляторов поля и особенности фотонной статистики возникающих неклассических состояний поля. Сжатие и корреляция квантовых фллуктуаций в системах нестационарных связанных осцилляторов рассматривались в последнее время в работах $[9,10]$. Нестационарная связь мод может возникать в процессе распространения когерентного света в нелинейной среде с показателем преломления, зависяшим от амплитуды поля. Результаты настояшей работы могут иметь, таким образом, приложения в нелинейных задачах квантовой оптики [11].

Целью нашей работы является изучение сжатия и возбуждения квантовых флуктуаций в коррелированных когерентных состояниях двумерного квантового осциллятора с параметрической связью координат конечной длительности. Модовые частоты предполагаются различными постоянными действительными числами. Предполагается, что координаты двумерного осциллятора связаны в течение ограниченного промежутка времени, причем частота связи является постоянной действительной величиной [12]. Гамильтониан осцилляторных мод с описанной выше нестационарной связью имеет вид

$$
\widehat{H}(t)=\frac{1}{2} \widehat{\mathbf{Q}} \mathbf{B} \widehat{\mathbf{Q}}
$$

* Московский физико-технический институт, Москва, Россия 
где $\widehat{\mathbf{Q}}=\left(\hat{p}_{1}, \hat{p}_{2}, \hat{q}_{1}, \hat{q}_{2}\right)-4$-столбец операторов импульсов $\left(\hat{p}_{i}\right)$ и координат $\left(\hat{q}_{i}\right)($ в квантовой оптике соответствуюших квадратурным компонентам двухмодового света), $\mathbf{B}(t)-$ $4 \times 4$-матрица:

$$
\mathbf{B}=\left(\begin{array}{cc}
\mathbf{I}_{2} & 0 \\
0 & \mathbf{B}_{2}
\end{array}\right), \quad \mathbf{B}_{2}=\left(\begin{array}{cc}
\omega^{2} & \lambda(t) \\
\lambda(t) & \omega^{-2}
\end{array}\right)
$$

где $\mathbf{I}_{2}$ - единичная $2 \times 2$-матрица. Гамильтониан (1) записан в безразмерных переменных таких, что энергия нормирована на $\hbar \sqrt{\omega_{1} \omega_{2}}$, где $\hbar$ - постоянная Планка, $\omega_{1,2}$ - частоты связанных мод, при этом $\omega^{2}=\omega_{1} / \omega_{2}$. Коэффициент связи $\lambda(t)$ полагается кусочно-постоянной функцией: $\lambda(0<t<T)=\omega_{0}^{2}, \lambda(t<0, t>T)=0, T$ - длительность связи мод (время измеряется в единицах $\left.\left(\omega_{1} \omega_{2}\right)^{-1 / 2}\right), \omega_{0}$ - безразмерная константа связи. В общем случае $\omega^{2} \neq 1$. Все частоты действительны, а массы осцилляторов выбраны равными единице. Введение нормальных координат канонической заменой, сохраняюшей коммутационные соотношения динамических переменных $\left[\widehat{Q}_{a}, \widehat{Q}_{b}\right]=-i \Sigma_{a b}$ $(a, b=1,2,3,4)$, где

$$
\boldsymbol{\Sigma}=\left(\begin{array}{cc}
0 & \mathbf{I}_{2} \\
-\mathbf{I}_{2} & 0
\end{array}\right)
$$

оказывается невозможным при $\omega^{2} \neq 1$, и поэтому задача будет решаться в исходных переменных.

2. Данная квантово-механическая модель является точно решаемой методом линейных интегралов движения $[13,14]$. Известно, что сушествование унитарного оператора эволюции системы делает возможным построение $2 N$ линейных интегралов движения $\widehat{\mathbf{p}}_{0}(t), \widehat{\mathbf{q}}_{0}(t)$ (в нашем случае число степеней свободы $N=2$ ) посредством канонического преобразования динамических переменных

$$
\hat{I}(t)=\left(\begin{array}{c}
\widehat{\mathbf{p}}_{0}(t) \\
\widehat{\mathbf{q}}_{0}(t)
\end{array}\right)=\boldsymbol{\Lambda}(t) \widehat{\mathbf{Q}}
$$

не меняюшего коммутационных соотношений $\left[\hat{I}_{a}, \hat{I}_{b}\right]=\left[\widehat{Q}_{a}, \widehat{Q}_{b}\right]$, так что $\boldsymbol{\Lambda}^{-1}=-\boldsymbol{\Sigma} \tilde{\boldsymbol{\Lambda}} \boldsymbol{\Sigma}$ (“тильда" означает транспонирование матрицы). Физический смысл интегралов движения (2) заключается в том, что они описывают $2 N$ начальных значений импульсов и координат системы. Согласно теореме Стоуна-фон Неймана [15] $\widehat{\mathbf{p}}_{0}(t)$ и $\widehat{\mathbf{q}}_{0}(t)$ образуют полный набор интегралов движения данной квантовой системы [13]: любая функция операторов $\widehat{\mathbf{p}}_{0}(t), \widehat{\mathbf{q}}_{0}(t)$ оказывается интегралом движения. Необходимым и достаточным условием инвариантности операторов $\widehat{\mathbf{p}}_{0}, \widehat{\mathbf{q}}_{0}$ является эволюция $\boldsymbol{\Lambda}$-матрицы согласно матричному уравнению

$$
\dot{\Lambda}=\Lambda \Sigma B
$$

с начальным условием $\boldsymbol{\Lambda}(0)=\mathbf{I}_{4}$, где $\mathbf{I}_{4}-$ единичная $4 \times 4$-матрица. Система $(3)$ с кусочно-постоянным коэффициентом связи имеет решение

$$
\boldsymbol{\Lambda}(t \leq T)=\mathbf{T}_{+} \otimes \boldsymbol{\Xi}_{+}-\mathbf{T}_{-} \otimes \boldsymbol{\Xi}_{-},
$$


где $\otimes$ означает тензорное произведение вспомогательных матриц

$$
\mathbf{T}_{ \pm}=\left(\begin{array}{cc}
\cos \left(\Omega_{ \pm} t\right) & \Omega_{ \pm} \sin \left(\Omega_{ \pm} t\right) \\
-\Omega_{ \pm}^{-1} \sin \left(\Omega_{ \pm} t\right) & \cos \left(\Omega_{ \pm} t\right)
\end{array}\right), \quad \boldsymbol{\Xi}_{ \pm}=\frac{1}{a_{+}-a_{-}}\left(\begin{array}{cc}
a_{ \pm} & 1 \\
1 & -a_{\mp}
\end{array}\right)
$$

выраженных через параметры $\omega_{ \pm}^{2}=\omega^{2} \pm \omega^{-2}, \Omega_{ \pm}^{2}=\frac{1}{2}\left(\omega_{+}^{2} \pm\left(\omega_{-}^{4}+4 \omega_{0}^{4}\right)^{\frac{1}{2}}\right), a_{ \pm}=$ $\left(\Omega_{ \pm}^{2}-\omega^{-2}\right) / \omega_{0}^{2}, a_{+} a_{-}=-1$. Матрицы $\boldsymbol{\Xi}_{ \pm}$удовлетворяют соотношениям $\boldsymbol{\Xi}_{ \pm} \mathbf{B}_{2}=$ $\Omega_{ \pm}^{2} \boldsymbol{\Xi}_{ \pm}$и обладают свойствами $\boldsymbol{\Xi}_{ \pm}^{2}= \pm \boldsymbol{\Xi}_{ \pm}$и $\boldsymbol{\Xi}_{+} \boldsymbol{\Xi}_{-}=\mathbf{0}$. Величины $\Omega_{ \pm}$совпадают с собственными значениями уравнений Гамильтона для классического двумерного связанного осциллятора с гамильтонианом (1). В условиях сильной связи $\left(\omega_{0}^{2}>1\right)$ классический двумерный связанный осциллятор имеет неположительно-определенную потенциальную энергию $\left(\operatorname{det} \mathbf{B}_{2}<0\right)$ и является неустойчивой системой. Собственное значение $\Omega_{-}$при этом чисто мнимое, что приводит к неустойчивому поведению элементов $\boldsymbol{\Lambda}$-матришы канонического преобразования (2). В противоположном случае слабой связи классическая система совершает финитное движение, собственные значения задачи действительны и элементы $\boldsymbol{\Lambda}$-матрицы ограничены.

3. Матрища дисперсий и ковариаций импульсов и координат

$$
\sigma_{Q}=\frac{1}{2}\left\langle\widehat{Q}_{\alpha} \widehat{Q}_{\beta}+\widehat{Q}_{\beta} \widehat{Q}_{\alpha}\right\rangle-\left\langle\widehat{Q}_{\alpha}\right\rangle\left\langle\widehat{Q}_{\beta}\right\rangle=\left(\begin{array}{cc}
\sigma_{p p} & \sigma_{q p}^{T} \\
\sigma_{q p} & \sigma_{q q}
\end{array}\right)
$$

- действительная симметричная положительно-определенная $4 \times 4$-матрища с элементами, подчиняюшимися определенным ограничениям (обобшенным соотношениям неопределенности [14]).

Предполагается, что при $t<0$ квантовое состояние рассматриваемой модели является когерентным состоянием [16] $\left|\alpha_{1}\right\rangle\left|\alpha_{2}\right\rangle$ (или как частный случай вакуумным состоянием $|0\rangle|0\rangle)$ несвязанных мод с матрицей дисперсий

$$
\sigma_{Q}(t \leq 0)=\left(\begin{array}{cc}
\mathbf{E}_{p} & \mathbf{0} \\
\mathbf{0} & \mathbf{E}_{q}
\end{array}\right)
$$

с диагональными блоками

$$
\mathbf{E}_{p}=\frac{1}{2}\left(\begin{array}{cc}
\omega & 0 \\
0 & \omega^{-1}
\end{array}\right), \quad \mathbf{E}_{q}=\frac{1}{2}\left(\begin{array}{cc}
\omega^{-1} & 0 \\
0 & \omega
\end{array}\right)
$$

Для вычисления $\sigma_{Q}(t)$ используется матрица дисперсий интегралов движения $\sigma_{I}=$ $\frac{1}{2}\left\langle\hat{I}_{a} \hat{I}_{b}+\hat{I}_{b} \hat{I}_{a}\right\rangle-\left\langle\hat{I}_{a}\right\rangle\left\langle\hat{I}_{b}\right\rangle$. Средние значения интегралов не зависят от времени, так что $\sigma_{I}=\sigma_{I}(t=0)=\sigma_{Q}(t=0)$. Поскольку $\widehat{Q}_{a} \widehat{Q}_{b}=\left(\boldsymbol{\Lambda}^{-1}\right)_{a i} \hat{I}_{i} \hat{I}_{j}\left(\boldsymbol{\Lambda}^{-1}\right)_{j b}$, имеем

$$
\sigma_{Q}(0 \leq t \leq T)=\boldsymbol{\Lambda}^{-1}(t) \sigma_{I}\left(\boldsymbol{\Lambda}^{T}(t)\right)^{-1}
$$




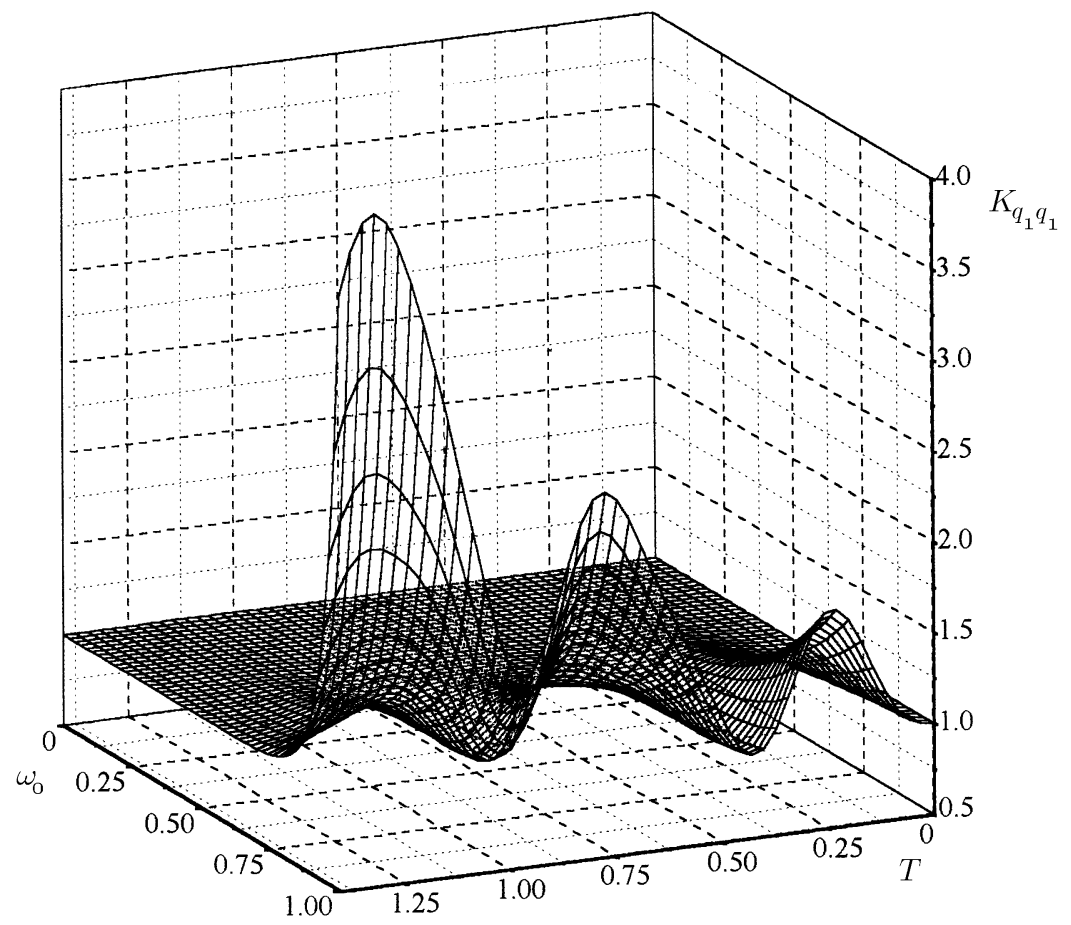

Рис. 1. Нормированная дисперсия координаты $K_{q_{1} q_{1}}$.

Отсюда непосредственно следуют выражения для $2 \times 2$-блоков матрицы дисперсий

$$
\begin{aligned}
\sigma_{p p}(t \leq T)= & \sum_{\sigma= \pm}\left(\frac{\sin ^{2}\left(\Omega_{\sigma} t\right)}{\Omega_{\sigma}^{-2}} \mathbf{A}_{q \sigma}+\cos ^{2}\left(\Omega_{\sigma} t\right) \mathbf{A}_{p \sigma}\right)- \\
& -\left(\frac{\sin \left(\Omega_{+} t\right) \sin \left(\Omega_{-} t\right)}{\Omega_{+}^{-1} \Omega_{-}^{-1}}-\cos \left(\Omega_{+} t\right) \cos \left(\Omega_{-} t\right)\right)\left(\mathbf{B}_{q}+\mathbf{B}_{q}^{T}\right), \\
\sigma_{q q}(t \leq T)= & \sum_{\sigma= \pm}\left(\frac{\sin ^{2}\left(\Omega_{\sigma} t\right)}{\Omega_{\sigma}^{2}} \mathbf{A}_{p \sigma}+\cos ^{2}\left(\Omega_{\sigma} t\right) \mathbf{A}_{q \sigma}\right)- \\
& -\left(\cos \left(\Omega_{+} t\right) \cos \left(\Omega_{-} t\right)-\frac{\sin \left(\Omega_{+} t\right) \sin \left(\Omega_{-} t\right)}{\Omega_{+} \Omega_{-}}\right)\left(\mathbf{B}_{q}+\mathbf{B}_{q}^{T}\right), \\
\sigma_{q p}(t \leq T)= & \sum_{\sigma= \pm} \frac{1}{2} \sin \left(2 \Omega_{\sigma} t\right)\left(\Omega_{\sigma}^{-1} \mathbf{A}_{p \sigma}+\Omega_{\sigma} \mathbf{A}_{q \sigma}\right)+ \\
& +\left(\frac{\sin \left(\Omega_{-} t\right) \cos \left(\Omega_{+} t\right)}{\Omega_{-}^{-1}}-\frac{\sin \left(\Omega_{+} t\right) \cos \left(\Omega_{-} t\right)}{\Omega_{+}}\right) \mathbf{B}_{q}+ \\
& +\left(-\frac{\sin \left(\Omega_{-} t\right) \cos \left(\Omega_{+} t\right)}{\Omega_{-}}+\frac{\sin \left(\Omega_{+} t\right) \cos \left(\Omega_{-} t\right)}{\Omega_{+}^{-1}}\right) \mathbf{B}_{q}^{T},
\end{aligned}
$$

где $\mathbf{A}_{q \pm}=\Xi_{ \pm} \mathbf{E}_{q} \Xi_{ \pm}, \mathbf{A}_{p \pm}=\Xi_{ \pm} \mathbf{E}_{p} \Xi_{ \pm}, \mathbf{B}_{q}=\Xi_{+} \mathbf{E}_{q} \Xi_{-}, \mathbf{B}_{p}=\Xi_{+} \mathbf{E}_{p} \Xi_{-}=-\mathbf{B}_{q}$. 


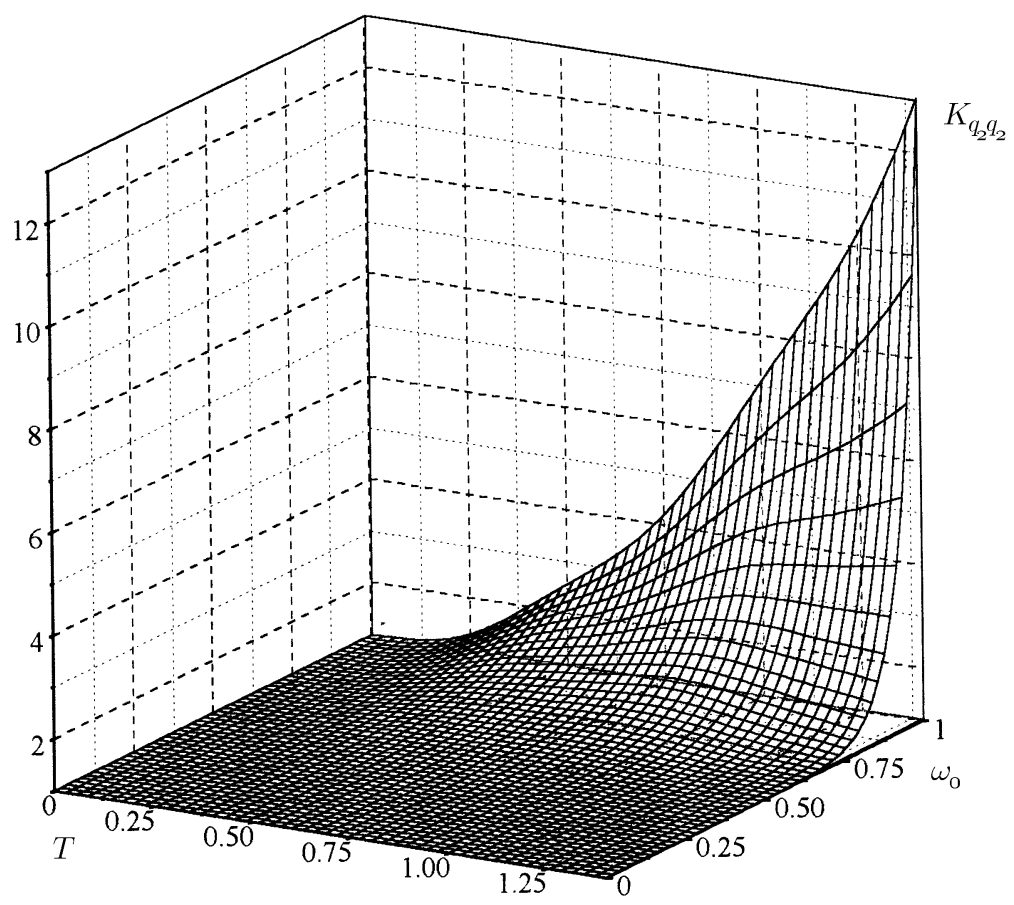

Рис. 2. Нормированная дисперсия координаты $K_{q_{2} q_{2}}$.

В процессе эволюции согласно уравнению Шредингера с квадратичным нестационарным гамильтонианом (1) состояние двумерного осциллятора $\left|\alpha_{1}, \alpha_{2}, t\right\rangle$ остается двумерным гауссовым пакетом, описываемым функцией Вигнера [17]

$$
W(\mathbf{Q}, t)=\left(\operatorname{det} \sigma_{Q}(t)\right)^{-\frac{1}{2}} \exp \left(-\frac{1}{2} \mathbf{Q} \sigma_{Q}^{-1} \mathbf{Q}\right)
$$

где двумерному чистому гауссову (когерентному) состоянию $\left|\alpha_{1}, \alpha_{2}, t\right\rangle$ соответствует минимизированное соотношение неопределенности [17] $\operatorname{det} \sigma_{Q}(t)=\operatorname{det} \sigma_{Q}(t=0)=$ $\left(\frac{1}{4}\right)^{2}$. Здесь 4 -мерный вектор $\mathbf{Q}=\left(p_{1}, p_{2}, q_{1}, q_{2}\right)$ образован вейлевскими символами операторов импульсов $\left(p_{i}\right)$ и координат $\left(q_{i}\right)$. Таким образом, временна́я эволюция состояния полностью определяется эволюцией матрицы дисперсий с блоками (6)-(8). Коэффициенты корреляции $\Gamma_{q_{i} p_{j}}=\sigma_{q_{i} p_{j}} / \sqrt{\sigma_{q_{i} q_{i}} \sigma_{p_{j} p_{j}}}$ определяют статистическую зависимость операторов координат и импульсов в данном состоянии. Поскольку коэффициенты корреляции, равные нулю в начальном когерентном состоянии несвязанных мод, становятся ненулевыми во все последуюшие моменты времени, мы говорим,что когерентное состояние связанных мод является "коррелированным".

При отсутствии связи мод матрица дисперсий эволюционирует во времени согласно уравнению, аналогичному (5):

$$
\sigma_{Q}(t>T)=\Lambda_{0}^{-1}(t-T) \sigma_{Q}(t=T)\left(\Lambda_{0}^{T}(t-T)\right)^{-1},
$$




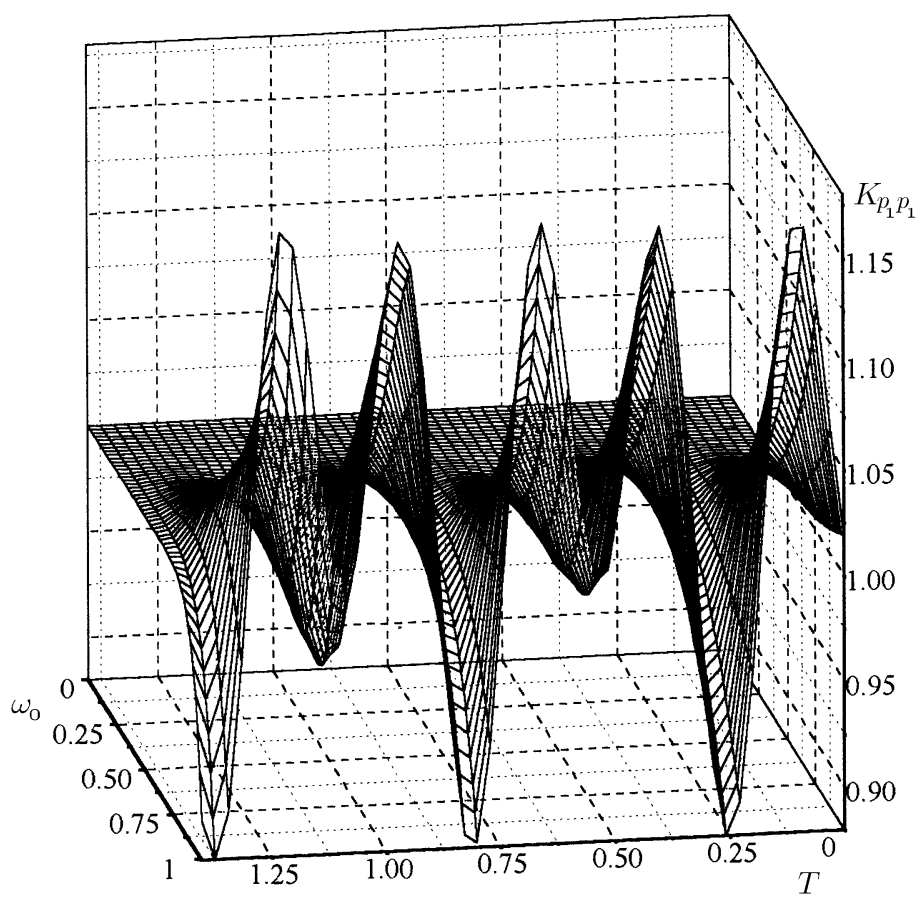

Рис. 3. Нормированная дисперсия импульса $K_{p_{1} p_{1}}$.

где $\boldsymbol{\Lambda}_{0}(t)$ есть $\boldsymbol{\Lambda}$-матрица (4) с $\omega_{0}=0$ (несвязанные моды). Явный вид $\sigma_{Q}(t>T)$ не приводится по причине его громоздкости.

На рис. 1-4 показаны дисперсии координат и импульсов $\left(\omega^{2}=5\right)$ как функции длительности $T$ и константы связи $\omega_{0}$, не превосходящей предела слабой связи $\left(\omega_{0}^{2}<1\right)$. Эти дисперсии нормированы на соответствующие дисперсии в начальном когерентном состоянии $K_{q_{1} q_{1}}=2 \omega \sigma_{q_{1} q_{1}}, K_{q_{2} q_{2}}=2 \omega^{-1} \sigma_{q_{2} q_{2}}, \quad K_{p_{1} p_{1}}=2 \omega^{-1} \sigma_{p_{1} p_{1}}, \quad K_{p_{2} p_{2}}=$ $2 \omega \sigma_{p_{2} p_{2}}$. Говорят, что состояние системы является сжатым, если дисперсии импульсов $\sigma_{p_{i} p_{i}}$ и/или координат $\sigma_{q_{i} q_{i}}$ оказываются меньше их значений в начальном когерентном состоянии. В этом смысле коррелированное когерентное состояние двумерного осциллятора в условиях слабой связи оказывается сжатым состоянием, т.к. при определенных значениях длительности и константы связи как $K_{p_{i} p_{i}}$, так и $K_{q_{i} q_{i}}$ могут оказаться меньше единицы (см. рис. $1,3,4)$. С другой стороны, при условии $T \omega_{0}>1$ возможно увеличение дисперсий квадратурных компонент по сравнению с их начальными значениями (это особенно заметно на рис. 2). При аналогичном условии (произведение константы связи на время наблюдения больше единицы) было обнаружено увеличение дисперсии числа фотонов для параметрического бозе-осциллятора [6, 7].

В условиях сильной связи можно получить асимптотический вид блоков (6)-(8) матрицы дисперсий при $\omega_{0} \gg \omega, \omega_{0} \gg \omega^{-1}$, учитьвая, что $\Omega_{+} \approx \omega_{0}, \Omega_{-} \approx i \omega_{0}$, и пренеб- 


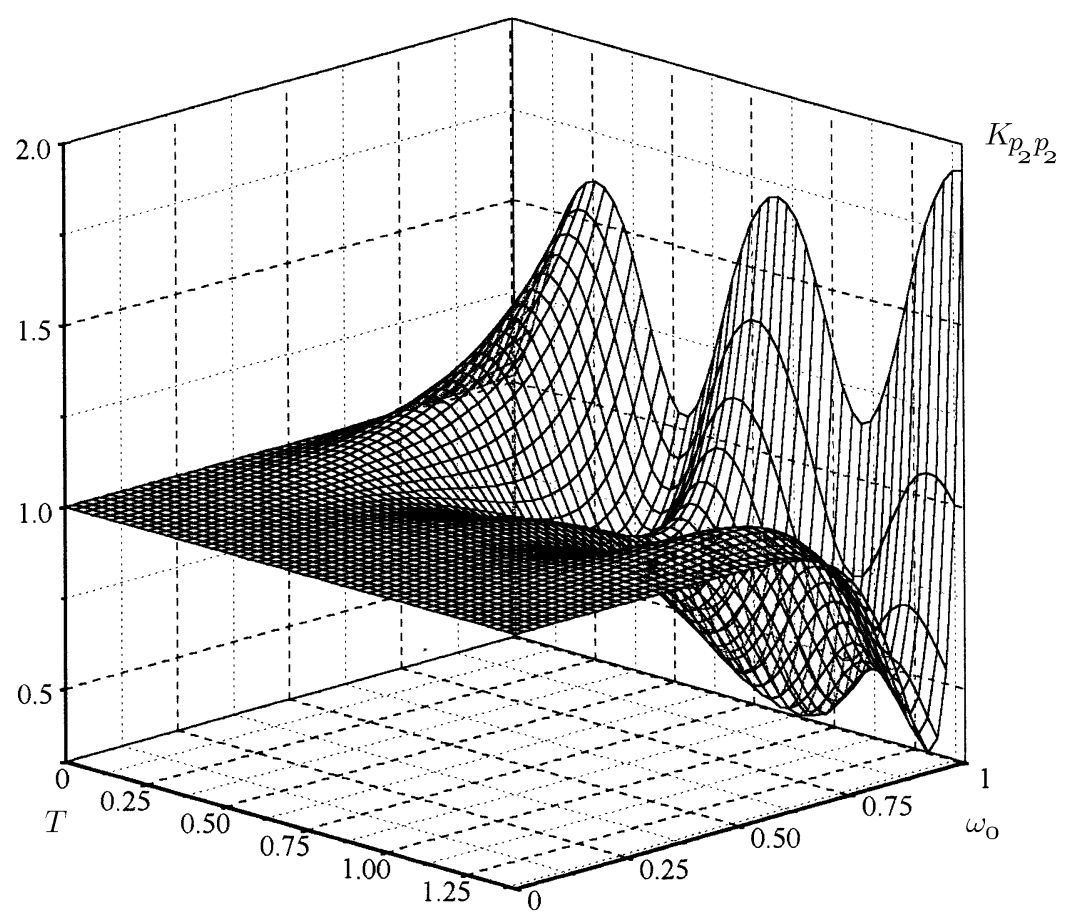

Рис. 4. Нормированная дисперсия импульса $K_{p_{2} p_{2}}$.

регая членами, растушими медленнее $e^{2 \omega_{0} t}$. В этом случае имеем

$$
\sigma_{p p}(t \leq T) \approx \frac{1}{32} \omega_{0}^{2}\left(\omega^{-1}+\omega\right) e^{2 \omega_{0} t}\left(\begin{array}{cc}
1 & -1 \\
-1 & 1
\end{array}\right)
$$

причем $\sigma_{q q}=\omega_{0}^{-2} \sigma_{p p}, \sigma_{p q}=-\omega_{0}^{-1} \sigma_{p p}$. В условиях сильной связи происходит сильное возбуж дение квантовых флуктуаций двумерного квантового осциллятора, что выражается в экспоненциальном временно́м росте их дисперсий (9). Заметим, что при этом состояние осциллятора остается сильно коррелированным $\left(\Gamma_{p_{i}} q_{j} \neq 0\right)$. Понятно, что в условиях сильной связи мод невозможно образование связанного состояния в двумерном потенциале классического связанного осциллятора (потенциальная энергия неположительно определена), а это значит, что система совершает инфинитное движение в своем фазовом пространстве, что и выражается на квантовом языке в экспоненциально неустойчивом поведении дисперсий и ковариаций импульсов и координат.

Итак, показано, что в зависимости от условий связи мод поведение квантовых флуктуаций в коррелированном когерентном состоянии двумерного квантового осциллятора может иметь принципиально разный характер: сжатие в условиях слабой связи и возбуждение и сильная корреляция при сильной связи мод.

Один из авторов (С. Ю.К.) благодарит Соросовскую образовательную программу (ISSEP) за поддержку (Соросовская стипендия для аспирантов, грант № a96-1913). 


\section{Список литературы}

[1] C. M. Caves, B.L Schumaker. Phys. Rev. A. 1985. V. 31. P. 3068; B. L. Schumaker, C. M. Caves. Phys. Rev. A. 1985. V. 31. P. 3093; S. M. Barnett, P. L. Knight. J. Opt. Soc. Amer. B. 1985. V. 2. P. 467.

[2] O. Castaños, R. Lopez-Pena, V. I. Man'ko. Phys. Rev. A. 1994. V. 50. P. 5209.

[3] Squeezed States of the Electromagnetic Field. J. Opt. Soc. Amer. B. 1987. V. 4. № 10.

[4] D.F. Walls. Nature. 1983. V. 306. P. 141.

[5] V. V. Dodonov, E. V. Kurmyshev, V.I. Man'ko. Phys. Lett. A. 1980. V. 79. P. 150.

[6] Л.А. Сюракшина, В.С. Ярунин. ТМФ. 1992. Т. 92. С. 158.

[7] L. A. Siurakshina, V.S. Yarunin. Phys. Lett. A. 1992. V. 164. P. 167.

[8] E. A. Kochetov, V.S. Yarunin. Physica Scripta. 1995. V. 51. P. 46.

[9] C. F. Lo. Phys. Lett. A. 1992. V. 162. P. 299.

[10] Leehwa Yeh, O. V. Man'ko. Phys. Lett. A. 1994. V. 189. P. 268.

[11] L. A. Lugiato, A. Gatti. Phys. Rev. Lett. 1993. V. 70. P. 3868.

[12] В. В. Додонов, О. В. Манько, В. И. Манько. Тр. ФИАН. 1989. Т. 191. С. 224.

[13] И. А. Малкин, В. И. Манько. Динамические симметрии и когерентные состояния квантовых систем. М.: Наука, 1979. C.72; I. A. Malkin, V. I. Man'ko. Phys. Lett. A. 1970. V. 32. P. 243.

[14] В. В. Додонов, В. И. Манько. Тр. ФИАН. 1987. Т. 183. С. 182.

[15] P. Рихтмайер. Принципы современной математической физики. М.: Мир, 1982. Т. 1. С. 362; J. von Neuman. Math. Annal. B. 1931. V. 104. P. 570.

[16] R. J. Glauber. Phys. Rev. 1963. V. 131. P. 2766.

[17] V. V. Dodonov, O. V. Man'ko, V.I. Man'ko. Phys. Rev. A. 1994. V. 50. P. 813.

Поступила в редакцию 16.XII.1996 г.

\section{E. Veisman, S. Yu. Kalmykov CORRELATED COHERENT STATES OF TWO-DIMENSIONAL QUANTUM OSCILLATOR WITH NONSTATIONARY MODE COUPLING}

The phenomena of squeezing and correlation of quantum fluctuations in the correlated coherent states of two oscillatory modes with a parametric coupling of coordinates are investigated. Under the conditions of strong mode coupling both the excitation and strong correlation of quantum fluctuations are discovered. The matrix of dispersions and covariances of dynamical variables is found explicitly with the use of the method of linear quantum integrals of motion. 